Supporting Information for

\title{
Microfluidic production of cell-laden microspheroidal hydrogels with different geometric shapes
}

\author{
Yuan Tian, Elizabeth A. Lipke*
}

212 Ross Hall, Auburn University, AL, 36849

Number of pages: 7

Number of figures: 1

Number of tables: 1 


\section{S1. PEGDA and PEG-fibrinogen synthesis.}

Poly(ethylene glycol) diacrylate (PEGDA) was synthesized as described previously. ${ }^{1}$ Briefly, poly(ethylene glycol) (PEG, 10kDa; Sigma-Aldrich) was reacted with acryloyl chloride (molar ratio 1:4; Alfa Aesar) in anhydrous dichloromethane (Acros Orgnics) with triethylamine (molar ratio 1:2; Sigma-Aldrich) under argon overnight at room temperature. The resulting PEGDA was purified by phase separation using $1.5 \mathrm{M} \mathrm{K}_{2} \mathrm{CO}_{3}$ (Fisher Scientific). The organic phase containing PEGDA was dried with anhydrous $\mathrm{MgSO}_{4}$ (Fisher Scientific). The PEGDA was then precipitated by cold diethyl ether (Sigma-Aldrich), filtered, dried, and stored under argon at $-20{ }^{\circ} \mathrm{C}$. The degree of acrylation was characterized by ${ }^{1} \mathrm{H}$ NMR.

PEG-fibrinogen was synthesized by following a previously published method. ${ }^{2}$ In brief, fibrinogen (Type I-S; Sigma-Aldrich) was dissolved in $8 \mathrm{M}$ urea (Sigma-Aldrich) phosphate buffered saline (PBS) solution to a final concentration of $7 \mathrm{mg} / \mathrm{mL}$. Tris(2-carboxy ethyl) phosphine (Acros Organics) was added to the solution, and the $\mathrm{pH}$ was then adjusted to 8. PEGDA was dissolved in urea-PBS to a final concentration of $280 \mathrm{mg} / \mathrm{mL}$ and then slowly added to fibrinogen solution to react for 3 hours in dark at room temperature. The resulting PEG-fibrinogen was precipitated with acetone and dissolved in urea-PBS again. The product was dialyzed in PBS for 24 hours in dark at $4^{\circ} \mathrm{C}$ and stored at $-80^{\circ} \mathrm{C}$. Protein concentration was measured using a Pierce BCA protein assay kit (Thermo Fisher). 


\section{S2. Microfluidic device fabrication and microfluidic platform setup.}

For microfluidic device fabrication, a molding technique was employed where a 3D printed jig was used to hold the molds. The jig with molds was then fixed on a glass surface using binder clips. Poly(dimethylsiloxane) (PDMS; Sylgard 184 silicone elastomer kit, Dow Corning) was poured into the jig, degassed by vacuum, and cured at $70^{\circ} \mathrm{C}$ for 2 hours. The molds were then removed. The PDMS microfluidic device was sterilized by sonicating in $70 \%$ ethanol before and after each use.

To produce hydrogel microspheroids using the microfluidic platform, hydrogel precursor solution was first prepared by mixing PEG-fibrinogen solution with $0.1 \%$ (w/v) of Pluronic F68 (Sigma-Aldrich), $0.1 \mathrm{mM}$ of Eosin Y photoinitiator (Fisher Scientific), 1.5\% (v/v) triethanolamine (Acros Organics), and $0.39 \%(\mathrm{v} / \mathrm{v})$ of $\mathrm{N}$-vinylpyrrolidone (Sigma-Aldrich). For cell-laden microspheroids production, cells were detached from the tissue culture flasks, centrifuged, and resuspended in hydrogel precursor solution to desired cell density.

Microspheroid production was conducted in a biosafety cabinet to keep the process clean. The microfluidic device had two inlets and one outlet where the hydrogel precursor solution was pumped in from the top inlet, and the mineral oil was pumped in from the bottom inlet. Both flow rates were controlled separately by syringe pumps. Microspheroids were formed at the junction of the microfluidic device due to emulsification and then photocrosslinked by a high intensity visible light source $\left(2.8 \mathrm{~W} / \mathrm{cm}^{2}\right.$; Prior Lumen 200$)$. The microspheroids were washed down at the end of the outlet channel using a third syringe pump and collected in a centrifuge tube. The residual mineral oil was removed through centrifugation. 


\section{S3. Regulation of relative velocity by narrowing ratio (NR).}

For volumetric flow rate V:

$$
V=u \pi\left(\frac{D}{2}\right)^{2}
$$

where $u$ is the flow velocity, and $\mathrm{D}$ is the diameter.

With this equation, the relative velocity of continuous phase (oil phase) to discrete phase (aqueous precursor solution) at the junction is shown as:

$$
\begin{gathered}
u_{C J}-u_{D J}=\left(\frac{V_{C}}{D_{J}^{2}}-\frac{V_{D}}{D_{J}^{2}}\right) \times \frac{4}{\pi} \\
=\frac{u_{C O} D_{O}^{2}}{D_{J}^{2}}-\frac{u_{D O} D_{O}^{2}}{D_{J}^{2}} \\
=\left(u_{C O}-u_{D O}\right) \frac{1}{N R^{2}} \\
\frac{u_{C J}-u_{D J}}{u_{C O}-u_{D O}}=\frac{1}{N R^{2}}
\end{gathered}
$$

where the subscripts $\mathrm{C}, \mathrm{D}, \mathrm{J}$, and o stand for continuous phase, discrete phase, junction, and outlet

channel, respectively. This equation shows that the NR determines the relative velocity at the junction while also accounting for the velocity in the outlet channel. 


\section{S4. Justification for not using Reynolds number as a parameter for predicting geometric shape in our microfluidic device.}

The range of Reynolds number for our microfluidic device is approximately $0.16-1.6$, which is at the lower end of the Reynolds number for most microfluidic devices (typically in the range of $10^{-1}$ to $10^{2}$ ). This low value is a result of our microfluidic devices having larger channel diameters than other systems and the necessary constraint on flow velocity in the outlet channel to ensure similar microspheroid light exposure time, which influences the hydrogel stiffness. Mechanical properties of the microenvironment have been shown to be critical for the fate of encapsulated cells.

Although Reynolds number is generally a useful parameter and potentially provides another metric for comparison between this system and other microfluidic platforms, it was not a particularly useful parameter in predicting geometric shapes in our microfluidic system. As mentioned above, the flow velocity in the outlet channel needs to be kept relatively constant for control over hydrogel stiffness. For capillary number $\mathrm{Ca}$ :

$$
C a=\frac{\mu \nu}{\gamma}
$$

where $\mu$ is the dynamic viscosity of continuous phase (oil phase), $v$ is the relative velocity of continuous phase (oil phase) to discrete phase (aqueous precursor solution) at the junction, and $\gamma$ is the interfacial tension (precursor solution-oil). When flow fraction is hold constant, the dynamic viscosity $\mu$ and interfacial tension $\gamma$ are constant. Ca now depends on the flow velocity at the junction. For incompressible fluid, the volumetric flow rate remains the same at the junction and in the outlet channel: 


$$
\begin{gathered}
V_{\text {Junciton }}=V_{\text {Outlet }} \\
v_{\text {Junction }} \pi\left(\frac{D_{\text {Junction }}}{2}\right)^{2}=v_{\text {Outlet }} \pi\left(\frac{D_{\text {Outlet }}}{2}\right)^{2} \\
v_{\text {Junction }}=v_{\text {Outlet }}\left(\frac{D_{\text {Outlet }}}{D_{\text {Junction }}}\right)^{2} \\
v_{\text {Junction }}=v_{\text {Outlet }} \frac{1}{N R^{2}}
\end{gathered}
$$

where $\mathrm{V}$ is the volumetric flow rate, $\mathrm{D}$ is the diameter, and $\mathrm{NR}$ is the narrowing ratio (as described in-depth in the manuscript).

Since the velocity in the outlet channel is held constant, the Ca which depends on the velocity at the junction is now determined by the NR of the microfluidic device.

However, for Reynold number:

$$
\begin{gathered}
\frac{R e}{C a}=\frac{\frac{\rho v D_{\text {Junction }}}{\mu}}{\frac{\mu \nu}{\gamma}}=\frac{\rho \gamma}{\mu^{2}} D_{\text {Junction }} \\
\operatorname{Re}=\frac{\rho \gamma}{\mu^{2}} D_{\text {Junction }} C a
\end{gathered}
$$

where $\rho$ is the fluid density. When $\mathrm{Ca}$ is held constant, the term

$$
\frac{\rho \gamma}{\mu^{2}}
$$

changes along with flow fraction.

But there is an extra term $D_{\text {Junction }}$. Based on our discussion in the manuscript, the junction diameter alone does not provide geometric control. Instead, the ratio of junction diameter to outlet diameter, which is the NR, is the determining factor. 
S5. The original images of T-junction in microfluidic device designs.
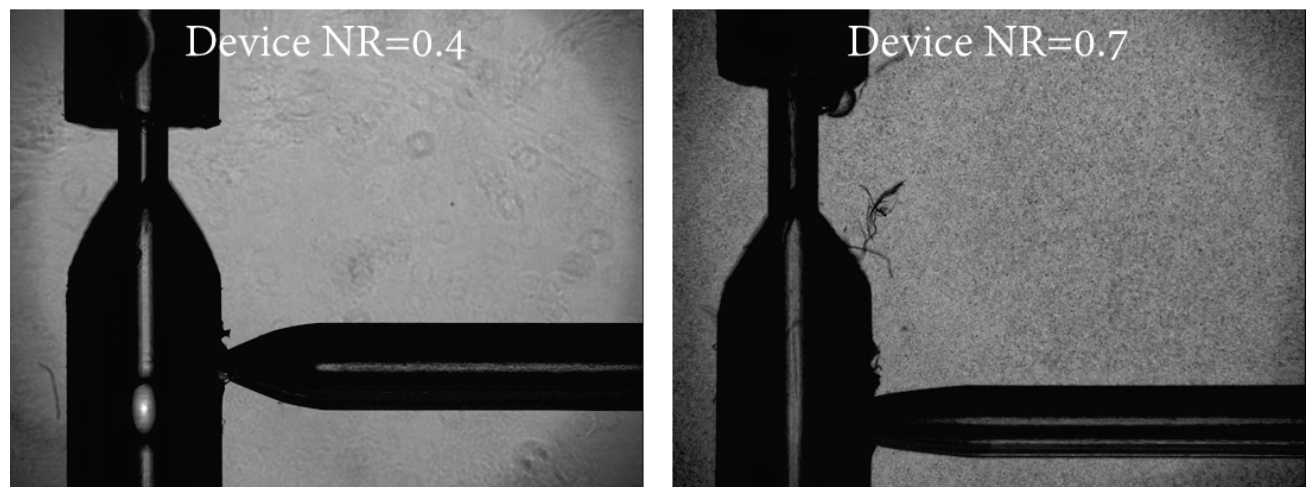

Figure S1-Sn. The original images of the T-junction in microfluidic devices with different narrowing ratios (NR).

S6. Examples of microfluidic device designs.

Table S1-Sn. Examples of microfluidic device designs.

\begin{tabular}{c|c|c|c|c|c}
\hline Device Design & $\mathrm{A}$ & $\mathrm{B}$ & $\mathrm{C}$ & $\mathrm{D}$ & $\mathrm{E}$ \\
\hline $\begin{array}{c}\text { Junction Size } \\
(\mu \mathrm{m})\end{array}$ & 220 & 330 & 450 & 506 & 533 \\
\hline $\begin{array}{c}\text { Outlet Size } \\
(\mu \mathrm{m})\end{array}$ & 740 & 895 & 950 & 760 & 716 \\
\hline $\begin{array}{c}\text { Narrowing } \\
\text { Ratio }\end{array}$ & 0.30 & 0.37 & 0.47 & 0.67 & 0.74 \\
\hline $\begin{array}{c}\text { Microspheroid } \\
\text { Geometry }\end{array}$ & Microspheres with flow fraction-dependent \\
diameters & $\begin{array}{c}\text { Microrods with flow fraction- } \\
\text { dependent axial ratios }\end{array}$ \\
\hline
\end{tabular}

\section{Reference}

1. DeLong, S. A.; Gobin, A. S.; West, J. L., Covalent immobilization of RGDS on hydrogel surfaces to direct cell alignment and migration. Journal of Controlled Release 2005, 109 (1-3), 139-148.

2. Almany, L.; Seliktar, D., Biosynthetic hydrogel scaffolds made from fibrinogen and polyethylene glycol for 3D cell cultures. Biomaterials 2005, 26 (15), 2467-2477. 